EPJ manuscript No.

(will be inserted by the editor)

\title{
Effects of anticipatory driving in a traffic flow model
}

\author{
Nils Eissfeldt ${ }^{1}$ and Peter Wagner ${ }^{2}$ \\ 1 ZAIK - Center for Applied Informatics, Universität zu Köln, 50931 Köln, Germany \\ ${ }^{2}$ Institute for Transportation Research, German Aerospace Center, 12489 Berlin, Germany
}

\begin{abstract}
Anticipation in traffic means that drivers estimate their leaders' velocities for future timesteps. In the article a specific stochastic car-following model with non-unique flow-density relation is investigated with respect to anticipatory driving. It is realized by next-nearest-neighbour interaction which leads to large flows and short temporal headways. The underlying mechanism that causes these effects is explained by the headways of the cars which organize in an alternating structure with a short headway following a long one, thereby producing a strong anti-correlation in the gaps or in the headways of subsequent cars. For the investigated model the corresponding time headway distributions display the short headways observed in reality. Even though these effects are discussed for a specific model, the mechanism described is in general present in any traffic flow models that work with anticipation.
\end{abstract}

PACS. 02.50.Ey Stochastic processes - 45.70.Vn Granular models of complex systems; traffic flow 89.75.Fb Structures and organization in complex systems

\section{Introduction}

'The basic mechanisms that are responsible for traffic flow breakdown are still not very well understood and discussed controversial [1,2,3,4. One reason for it is, that the microscopic models in use nowadays still have deficiencies, however it is not obvious which ones. Therefore it is not clear whether the mechanisms of breakdown displayed by a certain model have a counterpart in reality.

Sometimes, even the inner working of those models is not very well understood. This is true, e.g., for the models that work with so-called anticipation [5, 6]. Here, anticipation means that drivers estimate the velocity of preceding cars for future time steps. With respect to safe car motion this driving strategy avoids abrupt braking and therefore leads to a stabilization of the flow in dense traffic [5]. As a result, these models display small temporal headways that are similar to the ones observed in reality. Although the mechanism of stabilization of the flow seems to be necessary with respect to the reproduction of real-world traffic data [7, the changes in the model's dynamics in consequence of anticipatory driving are not known in detail. Therefore, it is the aim of this article to clarify the role of anticipation in microscopic traffic flow models.

This will be done for a certain well-known microscopic traffic flow model which is described in section 2 In this model, anticipation is introduced via next-nearest-neighbour interaction. The consequences on the model's dynamics is explored by simulation as well as analytical calculations (cf. section 3). Leaping ahead, it is stated that by virtue of anticipation the system organizes the headways of the cars in an alternating structure which allows for the observed small temporal headways. As will become clear from the discussion, most of the results found in the following should be at work in other models, too.

\section{A car-following model with anticipation}

As stated above, the effects of anticipation will be investigated using a specific car following-model. The model described in [8, 9,10] is used as reference model and is referred to as SKM in the following.

It is based on an approach by Gipps [11] and three basic assumptions, namely

- that vehicles move collision-free,

- not faster than a maximum velocity $v_{\max }$ and

- individual car acceleration $a$ and deceleration $b$ are bounded.

Based on the requirement of collision-freeness a safetycondition can be derived. Assume one car (driver-vehicle unit) with velocity $v$ is following another car (driving with velocity $\tilde{v}$ ) within a distance $g$. Here, $g$ is the free space between vehicles, i.e., the distance between the cars at positions $x, \tilde{x}$ minus the cars' length $l_{\text {car }}$. Safety, i.e., crashfree motion is guaranteed if

$$
d(v)+\tau v \leq d(\tilde{v})+g
$$

holds, with $d(v)$ being the braking distance needed to stop when driving with velocity $v$ and $\tau$ a finite reaction time. For braking with constant deceleration $b>0$, i.e., $-b \leq$ 


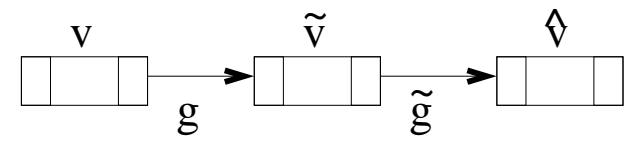

Fig. 1. Visualization of the variables $v, g, \tilde{v}, \tilde{g}, \hat{v}$. All cars are considered to have equal length $l_{\text {car }}$.

$d v / d t$ the braking distance is given by $d(v)=v^{2} /(2 b)$. The equation (11) then leads to

$$
v_{\text {safe }}(\tilde{v}, g)=-b \tau+\sqrt{b^{2} \tau^{2}+\tilde{v}^{2}+2 b g} .
$$

In order to complete the definition of the model's dynamics it is assumed that every car moves at the highest velocity compatible with the assumptions. Based on these assumptions an update scheme can be formulated in the manner of the well-known Nagel-Schreckenberg model (NaSch) 12.

The SKM is defined with continuous state variables $x, v$ and discrete timesteps $\Delta t$. In each timestep every car is updated after calculating its $v_{\text {safe }}$ according to the following scheme

$$
\begin{aligned}
v_{\text {des }} & =\min \left\{v_{t}+a \Delta t, v_{\text {safe }}, v_{\max }\right\} \\
v_{t+\Delta t} & =\max \left\{v_{\text {des }}-\eta \epsilon a, 0\right\} \\
x_{t+\Delta t} & =x_{t}+v_{t+\Delta t} \Delta t .
\end{aligned}
$$

The update (3) is done in parallel. The random fluctuation of strength $\eta \epsilon a$ is introduced to mimic deviations from the optimal driving strategy given by $v_{\text {safe }} . \eta$ is a random number uniformly distributed in the interval $[0,1]$ and the parameter $\epsilon$ determines the fluctuation strength in units of $a$.

Before introducing anticipation into the model it should be stated that the formulation of $v_{\text {safe }}$ in equation (2) differs from that given in [9]. The reason is mostly that the calculation of the update scheme (3) becomes more easy. However, due to its structure we could not find a proof for crash-freeness analytically (as is possible for the original formulation). But extensive simulations with $\tau \geq \Delta t^{1}$ neither gave a hint for collisions nor had we found a crucial difference in the model's dynamics.

We recall that in the SKM each car only takes into account the car in front to deduce its optimal driving strategy. It is common experience that such assumption is unrealistic, especially in dense traffic situations. In order to bring anticipation into the model the update scheme is modified by an intermediate step: Each driver predicts the worst-case strategy $v_{\text {anti }}$ her predecessor will choose in the next timestep. Assuming that there is a car in front of the predecessor within a distance $\tilde{g}$ driving with velocity $\hat{v}$ (see figure 1), then

$$
v_{\text {anti }}=\max \left\{\tilde{v}_{\text {des }}-\epsilon a, 0\right\}
$$

with

$$
\tilde{v}_{\mathrm{des}}=\min \left\{\tilde{v}+a, \tilde{v}_{\mathrm{safe}}(\hat{v}, \tilde{g}), v_{\max }\right\}
$$

1 This conditions simply states that safe driving is possible, if the "true" reaction time, i.e., one timestep, is smaller or equal to the reaction time each driver assumes.
The calculated $v_{\text {anti }}$ will then be used to determine the safe velocity. Therefore, the safety condition equation (11) is restated with the assumption that the leading car will choose $\tilde{v}_{t+\Delta t} \geq v_{\text {anti }}$ as driving strategy,

$$
d(v)+\tau v+\gamma_{c}(v, \tilde{v}) \leq d\left(v_{\mathrm{anti}}\right)+v_{\mathrm{anti}} \tau+g .
$$

The function $\gamma_{c}(v, \tilde{v})$ has been introduced to take into account "unexpected" fluctuations in the predecessor's driving behaviour. Then, the new expression equation (6) leads to a new expression for the safe velocity,

$$
\begin{aligned}
v_{\mathrm{safe}}= & -b \tau \\
& +\sqrt{b^{2} \tau^{2}+v_{\mathrm{anti}}^{2}+2 b\left(g+v_{\mathrm{anti}} \tau-\gamma_{c}(v, \tilde{v})\right)} .
\end{aligned}
$$

In the following,

$$
\gamma_{c}(v, \tilde{v})=\min \left\{v_{\text {anti }} \tau, g_{c}\right\},
$$

will be chosen where $g_{c}$ is constant. Since $g+v_{\text {anti }}-\gamma_{c}(v, \tilde{v})$ can be interpreted as an effective gap $g_{\text {eff }}$, where equation (8) forces $g_{\text {eff }} \geq g$. The idea of the effective gap is similar to the cellular-automaton model in [5] (BL-CA). The major difference is that in the modified SKM anticipation enters into the model by velocity and the effective gap (cf. equation (77) while in the BL-CA it does just via the latter.

Besides the new definition of $v_{\text {safe }}$ the update scheme (3) is used.

\section{The role of anticipation}

In this section, by means of computer simulations, the SKM with anticipation (SKA) will be compared to the original model. For this purpose, a fixed set of parameters is used, namely

$$
\begin{aligned}
& a=2 \mathrm{~m} / \mathrm{s}^{2} \quad b=8 \mathrm{~m} / \mathrm{s}^{2} \quad v_{\max }=35 \mathrm{~m} / \mathrm{s} \\
& \epsilon=1 \quad g_{c}=1 \quad l_{\text {car }}=7 \mathrm{~m}
\end{aligned}
$$

As time scale $\tau=\Delta t=1 \quad s$ is chosen. With respect to these parameters jam formation (wide moving jams) and stable high-flow states exist in the corresponding SKM 9 . [13] (cf. following subsection).

\section{Flow-density relation}

To get started both models were simulated using periodic boundary conditions, i.e., on a one-lane loop. In order to measure the flow-density relation the loop was initialized homogeneously at different global densities. After relaxation of the system mean density $\langle\rho\rangle$, mean velocity $\langle v\rangle$ and flow $\langle q\rangle$ were measured at a fixed location using $60 \mathrm{~s}$ intervals for sampling. The local density for a car $n$ passing the counting location is defined as

$$
\rho_{n}=1 /\left(g_{n}+l_{\text {car }}\right)
$$



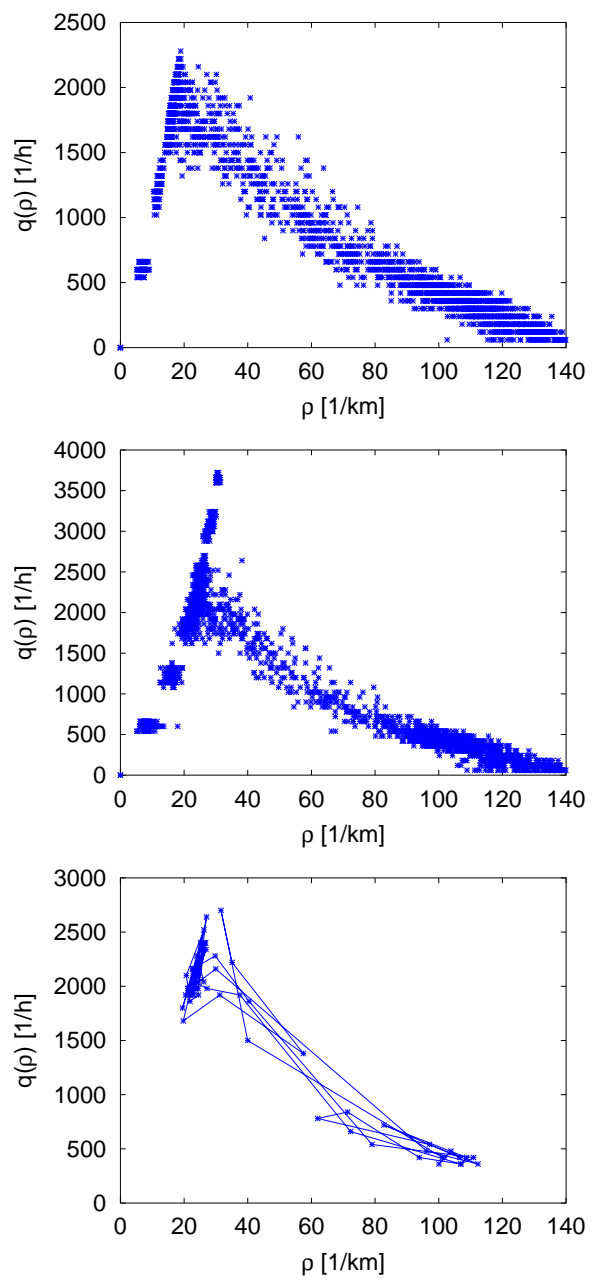

Fig. 2. Plotted is the "local" fundamental diagram for the SKM (top), SKA (middle) as function of the density, and for the SKA where the global density has been fixed to $\rho=$ $35 \mathrm{~km}^{-1}$ (bottom), a region where jammed and free flow coexist. Local means that the plot is obtained by mimicking a loop detector, and the time-averaging is done only over $60 \mathrm{~s}$.

Comparing the flow-density relations of the models (figure 21) they both display a high-flow state and a capacity drop at intermediate densities. The latter indicates slowto-start behaviour. Note that there is no explicit rule introducing this effect and it results from the the asymmetry in the randomization process for small speeds. As can be seen, this mechanism is not changed by the introduction of next-nearest-neighbour interactions. Moreover, the "optimized" driving strategy even leads to a stabilization of the high-flow branch towards higher densities compared to the SKM as already stated.

In the closed system the jam state co-exists with the free-flow state for densities $\rho \geq 20 \mathrm{~km}^{-1}$. Time-series at a fixed density in that regime therefore display free-flow and jammed states alternately (figure 2). At densities where the homogeneous free-flow state is unstable, small clusters of cars are generated due to intrinsic fluctuations. In the long run, this number of jams decreases until only one jam is left (figure 3i). Recalling the arguments of [9] the
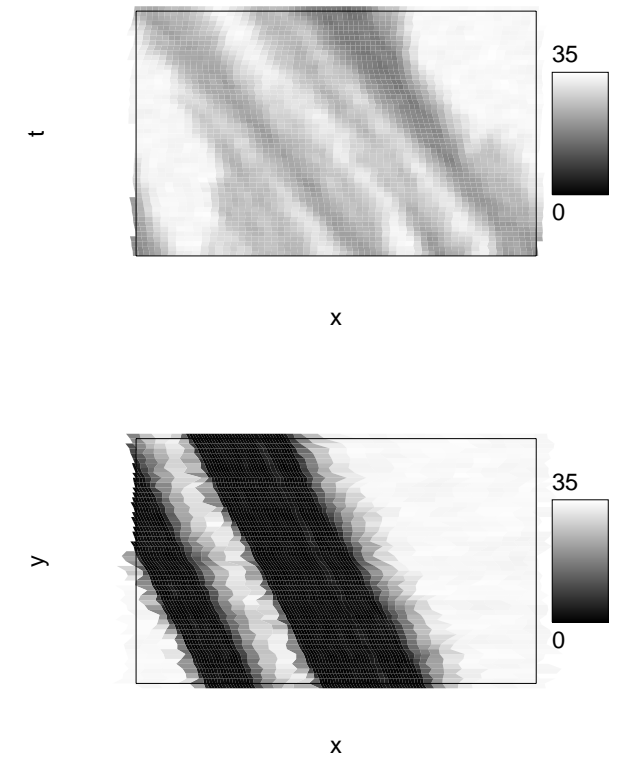

Fig. 3. Space-time-diagram for a typical evolution of the SKA. Each car is coloured by its current velocity in $\mathrm{m} / \mathrm{s}$. Initially, there are a lot of small jams (top) that coagulate into a double jam state (bottom). However, when waiting for a very long time, only one wide moving jam remains.

jamming transition in the SKA is a phase transition and one finds a phase-separated system at equilibrium using periodic boundary conditions.

In 13 a classification for stochastic traffic flow models is provided based on the breakdown mechanism. To be more precise, in the density regime where the jam solution co-exists with the high-flow state one distinguishes two classes. Models are said to have a "stable outflow" if intrinsic fluctuations are not able to trigger the transition from homogeneous flow to the congested phase. With respect to the chosen parameters, the SKM belongs to that class of models. In contrast, models like the VDR-model [14] display real metastability in that density regime and are said to have "unstable outflow". In figure 4 the waiting time until the first stopped car is found is shown versus the system's density. For each run a system with 5000 cars was initialised homogeneously. The values presented are means of 20 realisation per density. As can be seen, at $\rho_{c} \approx 31 \mathrm{~km}^{-1}$ this time diverges, i.e., homogeneous states corresponding to $\rho \leq \rho_{c}$ are stable. These state correspond to the high-flow branch in the flow-density relation (figure 21) and therefore, the SKA owns the same type of bistability 13 as the SKM does.

By virtue of the anticipatory driving strategy, the maximum attainable flow in the SKA is unrealistically high, which is a known feature for some extensions of the NaSchmodel as well [7. In that case the high flows only occur, if anticipatory driving as defined in [5] is switched on in, e.g. the VDR-model [14, without changing the model's parameters. 


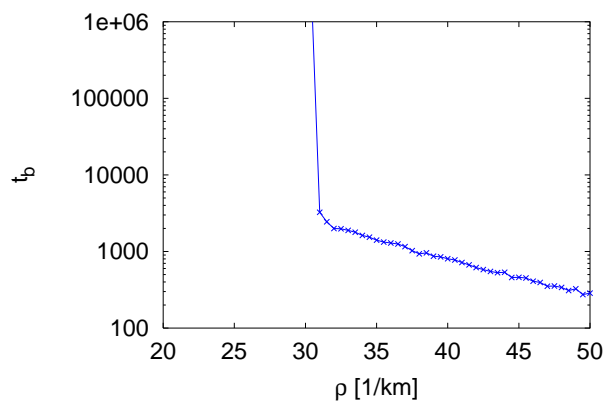

Fig. 4. The average break-down time $t_{\mathrm{b}}$ in seconds for the SKA. For any density, a system with 5000 cars has been initialized with the homogeneous state. After waiting for at most $10^{6}$ time-steps and repeating this for 20 different realizations, the average time to breakdown can be approximated.

Even though such states only appear for special initial conditions, i.e., highly ordered homogeneous configurations, modifications are necessary to use it in reasonable applications. However, it is a different question whether these flows can be attained in a realistic settings with macroscopic disturbances from lane-changing, on- and offramps etc. Additionally, by introducing a diversified driver behaviour also might lower the maximum flow to realistic values. For example this might be done is by using a distribution $p(\tau)$ for the parameter $\tau$ so that each driver has her individual $\tau_{i}$ drawn from that distribution. Another way of doing it is to increase $g_{c}$. Nevertheless, we will not take into account such problem since we concentrate at discussing the effects of anticipatory driving on the SKM.

Apart from these unrealistic high flows, it can be concluded that the all-over macroscopic properties of the SKA under periodic boundary conditions (i.e., the global fundamental diagram, the spontaneous jam formation or the existence of compact jams) are similar to the corresponding SKM.

\section{Time-headway distribution}

Several empirical studies have analyzed single vehicle data from counting loops 15, 16, 17, 18, 19. Such measurements provide information about the microscopic structure of traffic

streams. The investigation of the corresponding observables in stochastic traffic flow models can therefore justify their quality.

The time-headway is the microscopic analogue of the inverse flow. In real data, it is simply measured by the time-difference $t_{h}=t_{i-1}-t_{i}$ between the times of two cars passing the observer. Since this model has a timestep of $\Delta t=1 \mathrm{~s}$, a different approach has to be used to measure the time headway between two cars. This is done by using the relation

$$
t_{h}=g / v
$$

The closed loop still serves as the computer-experimental setup. It is initialized at different densities and the time-
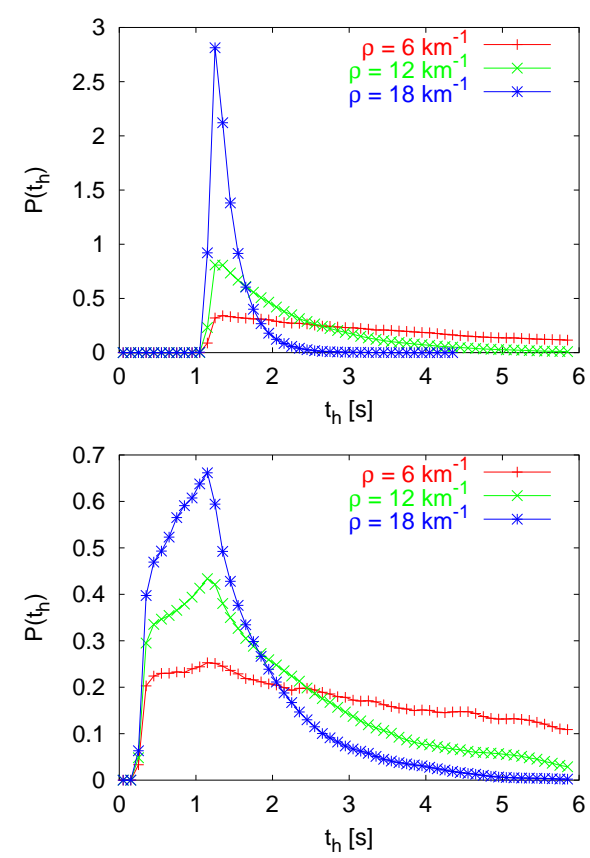

Fig. 5. Time headway distribution for the SKM (top) and SKA (bottom) under free-flow conditions.

headway distribution is measured after a sufficient relaxation time.

In figure 5 the time-headway distribution of the free flow phase at different densities is presented. From empirical investigation it is known that in free flow extremely small time-headways exist $\left(t_{h}^{\min } \approx 0.2 \mathrm{~s}\right)$. Moreover, the maximum of the distribution and its shape at short times are independent of the density [17,18,19].

Figure 5 (top) shows that the original SKM is not able to reproduce such small time-headways in the free flow phase. There exist a sharp cut-off at $t_{h} \approx \Delta t=1 \mathrm{~s}$, i.e., the model's dynamics leads to $v_{t+\Delta t} \leq g_{t}$. The maximum of the distributions is located at $t_{h} \approx 1.3 \mathrm{~s}$. Since in free flow $\langle v\rangle \approx 34 \mathrm{~m} / \mathrm{s}$ this corresponds to $\langle g\rangle \approx 41.5 \mathrm{~m}$, i.e., the SKM owns a fix point in its dynamics (for more details see next subsection)

In contrast, the time-headway distribution of the SKA shows a broader peak structure (figure $\mathbf{5}$ (bottom)) and headways noticeable smaller than $1 s$ exist, just as in empirical observations. Even more, the distribution at short times is independent of the density. However, the broadness of the peaks is not found in real-world observations. The occurrence of short time-headways stems from the introduction of the velocity anticipation. Drivers can optimize their gap to the leading vehicle since they have an idea about its future behaviour. Smaller gaps at $v \approx v_{\max }$ than in the SKM are therefore allowed.

Moreover, the broadness of the peaks indicates that in the SKA a range of gaps can be taken by cars driving at $v \approx v_{\max }$ in the free flow phase, i.e., there is no such strong fixed-point in the car-following dynamics as is in the SKM (leaping ahead, this results from the fact that in the SKA two consecutive cars share their common gap 

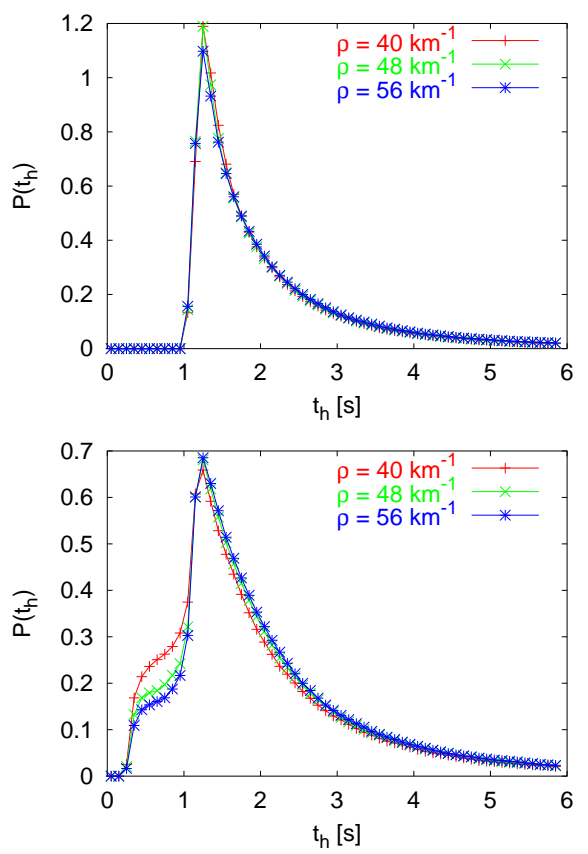

Fig. 6. Time headway distributions for the SKM (top) and SKA (bottom) in congested flow. Headways smaller than $1 s$ stem from the free flow state, their share decreases with increasing density.

$g+\tilde{g})$. With increasing density the peak in the distribution becomes more and more pronounced and is shifted towards smaller time-headways. The position of the peak correspond to the mean gap, given by the initial conditions, $\langle g\rangle=1 / \rho-l_{\text {car }}$. Moving towards higher densities along the free-flow branch of the flow-density relation the possible range of gaps between cars decreases.

Comparing the time-headway distributions in the congested state (figure 6), they are for both models almost independent of the density. The exponential decay of the distribution results from the fact that for large headways cars can be regarded as almost independent from each other, implying a Poissonian distribution. They have their maximum around $t_{h} \approx 1 \mathrm{~s}$ which agrees with empirical findings. Unlike reality the peak is fixed and not as broad.

From this we conclude that concerning the dynamics inside jams both models behave similarly.

The appearance of $t_{h} \leq 1$ in the case of the SKA is due to the experimental setup. In the closed loop the system is separated into two phases, one wide moving jam and a region of free-flow (figure 31). As demonstrated before, time-headways smaller than $1 s$ can be found in the freeflow phase. Therefore, cars that are not in the jammed state generate these time-headways. Since the number of cars in the free phase decreases with increasing density the weight of small time-headways also reduces.

\section{Optimal velocity curve}

Neglecting fluctuations, the optimal velocity curve (OVC) of both models can be derived analytically. This relation is

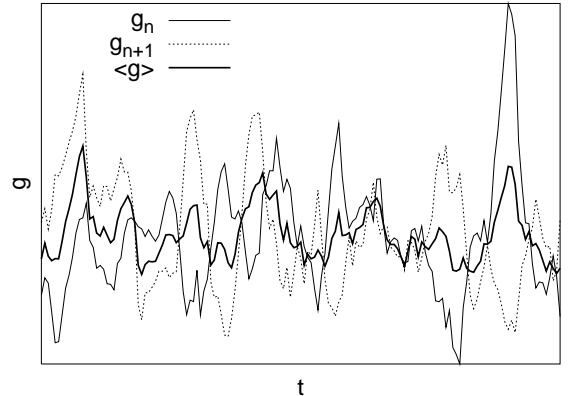

Fig. 7. Time series of the gaps between two consecutive cars in the modeled chain of cars in a typical simulation. The leading cars drives at $V=15 \mathrm{~ms}^{-1}$. The mean gap $\langle g\rangle=\left(g_{n}+g_{n+1}\right) / 2$ varies hardly, since the two cars share a common gap $\propto 1 / \rho$. The gaps of two consecutive cars are clearly anticorrelated.

helpful in order to characterize the microscopic structure of the traffic phases [17, 18, 19.

The OVC of the SKM results from its safety-condition (21), i.e.

$$
v_{\text {safe }}=-b \tau+\sqrt{b^{2} \tau^{2}+\tilde{v}^{2}+2 b g}=\tilde{v} .
$$

Equation (10) is solved by $\tilde{v}=g / \tau$, therefore the OVC of the SKM reads

$$
V_{\mathrm{opt}}^{\mathrm{SK}}(\rho)=\min \left\{\frac{1}{\tau}\left(\frac{1}{\rho}-\frac{1}{\rho_{\max }}\right), v_{\max }\right\} .
$$

From equation (11) it follows that $t_{h}^{\mathrm{sk}}=\tau$ for the deterministic case. The OVC asserts the results that timeheadways smaller than $\Delta t$ can not be modeled by the SKM since $\tau \geq \Delta t$ is required due to safety constraints and the stochasticity leads to a lowering of $\langle v\rangle$.

Regarding the SKA two cases have to be distinguished (cf. equation (8)). The OVC is derived from the condition

$$
\begin{aligned}
& \hat{v}=v_{\text {safe }}= \\
& -b \tau+\sqrt{b^{2} \tau^{2}+v_{\text {anti }}^{2}+2 b\left(g+v_{\text {anti }} \tau-\gamma_{c}\right)},
\end{aligned}
$$

with

$$
v_{\text {anti }}=-b \tau+\sqrt{b^{2} \tau^{2}+\hat{v}^{2}+2 b \tilde{g}} .
$$

. In the case of $v_{\text {anti }}>g_{c}$, i.e. $\gamma_{c}=g_{c}$, equation (12) is solved by $\hat{v} \tau=\left(g+\tilde{g}-g_{c}\right)$ and the OVC reads

$$
V_{\mathrm{opt}}^{\mathrm{f}}(\rho)=\min \left\{\frac{2}{\tau}\left(\frac{1}{\rho}-\frac{1}{\rho_{\max }}-\frac{g_{c}}{2}\right), v_{\max }\right\},
$$

If $v_{\text {anti }}<g_{c}$, i.e., $\gamma_{c}=v_{\text {anti }} \tau$ the known expression of the SKM follows,

$$
V_{\text {opt }}^{\mathrm{j}}(\rho)=V_{\text {opt }}^{\mathrm{SK}}, \quad v_{\text {anti }}<g_{c} .
$$

Therefore, in the high density regime, the SKA behaves like the SKM as already stated with respect to the flowdensity relation. 


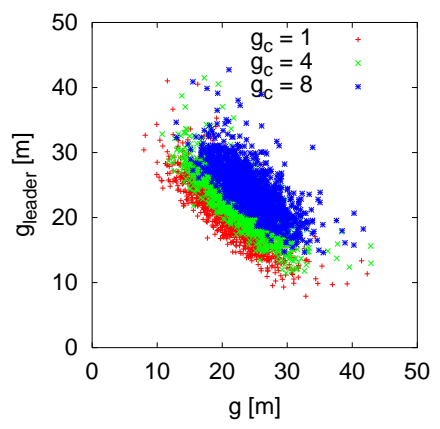

Fig. 8. The simulations performed with the stochastic version of the SKA and for different values of $g_{c}$ are in qualitative agreement with equation (23).

\section{Follow-the-leader behaviour}

Finally, we investigate the differences in the follow-theleader behaviour between the two models. For this purpose we use a chain of 1000 cars that follow the first car whose speed is fixed to $V \leq v_{\max }$. The system is initialized by all cars standing $\left(g_{i}=v_{i}=0\right)$. The zeroth car accelerates until the constant velocity $V$ is reached. Since then the system can be assumed to be stationary, quantities start to be measured once for the last car of the chain $x \geq$ $10000 \cdot V$. Before presenting the simulation results this set-up will be analysed more closely.

In the following just the deterministic case will be regarded. Then, all the speeds can be eliminated to yield an update equation just for the gaps. In order to keep the equations concise we adopt the following notation: If a quantity is labeled with a prime' it means timestep $t+\Delta t$, all others are to be taken at timestep $t$. For the same reason $\tau=1$ is used in the following.

Assuming a lead car driving at constant speed $v_{0}=$ $V$, the behaviour of the SKM is then determined by the equations

$$
\begin{aligned}
& v^{\prime}=v=-b+\sqrt{b^{2}+V^{2}+2 b g} \\
& g^{\prime}=g=g+V-v^{\prime}
\end{aligned}
$$

Equation (16) has a fixed-point at $v^{*}=V$ and $g^{*}=V$. Since this result can be expanded to the full chain of cars it follows that

$$
g_{n+1}=g_{n} .
$$

The lower index denotes the position $n$ of the car in the chain.

The result explains the independence of the peak in the time-headway distribution at low densities (cf. figure 5). Moreover, it follows that the stochasticity of the SKM is not able to destroy the fixed-point entirely. It is worth to say that the robustness of the fixed-point in continuous car-following models is hard to overcome and causes problems to model the synchronized state.

Now, the same situation is investigated for the SKA. Again, the lead car drives constantly with $v_{0}=V$ which is

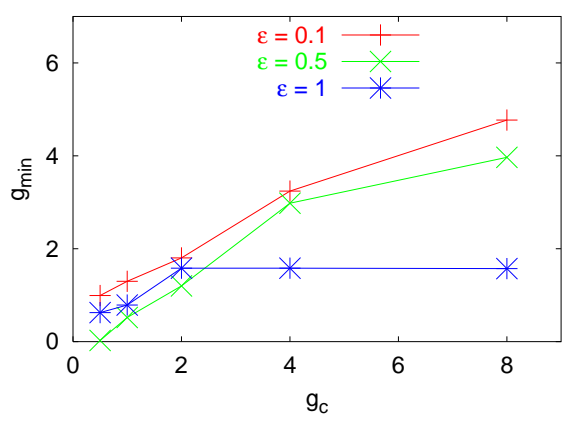

Fig. 9. Minimal gap found in simulations of the loop taking different values for $g_{c}([m])$ and $\epsilon$.

also $v_{\text {anti }}$ for the first following car. Regarding the deterministic case, that car then drives with $v_{1}^{*}=V$ and with constant headway $g_{1}^{*}=g_{c}$. This is because

$$
v_{1}^{\prime}=-b+\sqrt{(b+V)^{2}+2 b\left(g_{1}-g_{c}\right)}
$$

and

$$
g_{1}^{\prime}=g_{1}+V-v_{1}^{\prime},
$$

whose fixed-point $g_{1}^{\prime}=g_{1} \equiv g_{1}^{*}$ is just $g_{c}$.

For the second car this procedure can be carried out to give

$$
g_{2}^{\prime}=b+V+g_{2}-\sqrt{b^{2}+V^{2}+2 b g_{2}},
$$

where the stationary state of the first car $v_{1}^{*}=V$ and $g_{1}^{*}=g_{c}$ has been assumed. This equation has a simple fixed-point, namely $g_{2}^{*}=V$. Obviously $v_{2}^{*}=V$ holds alike.

For the third car the computation leads to

$$
\begin{aligned}
g_{3}^{\prime} & =b+V+g_{3} \\
& -\sqrt{b^{2}-2 b g_{c}+V^{2}+2 b g_{2}+2 b g_{3}},
\end{aligned}
$$

and $g_{3}^{*}=g_{c}, v_{3}^{*}=V$. Generalized, the latter equation reads

$$
\begin{aligned}
g_{n+1}^{\prime} & =b+V+g_{n+1} \\
& -\sqrt{b^{2}-2 b g_{c}+V^{2}+2 b g_{n}+2 b g_{n+1}},
\end{aligned}
$$

leading to the following expression for the stationary state $g_{n}^{\prime}=g_{n}, v_{n}=V$ :

$$
g_{n+1}=-g_{n}+g_{c}+V .
$$

This result, equation (23), shows that asymptotically the gaps of the $n$-th and $(n+1)$-th car are anti-correlated. In figure 8 time series $g_{n}$ vs. $g_{n+1}$ are displayed for different values of $g_{c}$. With increasing $g_{c}$ the corresponding line moves away from the origin, therefore the corresponding flow decreases.

The result helps to understand the plateau structure found in the time-headway distribution of the SKA at low densities (cf. figure 51). With respect to equation (23) the 

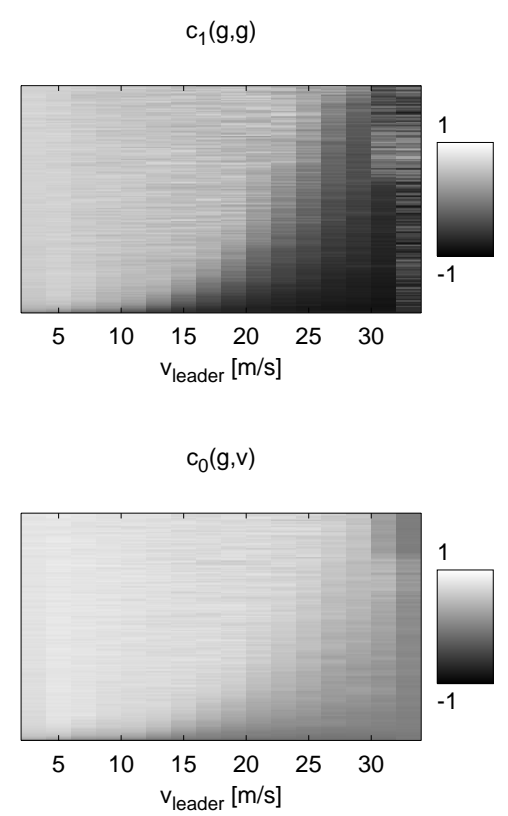

Fig. 10. Correlation functions $c_{1}(g, g)$ (top) and $c_{0}(g, v)$ (bottom) for the follow - the-leader experimental setup in the SKA. The correlations (value on the z-axis) have been calculated for each car in the chain, i.e. the value on the $y$-axis correspond to the car number $n$.

time-headways of two consecutive cars cover the boundary-points of the interval $\left[g_{c} / V, 1\right]$. Compared to the distribution of time headways measured in simulation the lower bound will hardly be reached due to stochasticity in the latter case. As in the SKM stochasticity is not able to destroy the fixed-point structure of the model, but two cars can exchange their role in that structure, i.e., two cars share a gap given by mean density but the share between $g_{n}$ and $g_{n+1}$ is not fixed. Figure 7 displays a time series of two cars sharing a common gap.

Before the correlation structure of the models is investigated in more detail, the role of $g_{c}$ is shown. Looking at equation (23) one could wonder why the anticorrelation does not lead to states with $g<0$.

Assume that a car $(n+1)$ has closed in on its predecessor $n$. Recalling equation (22), the gap $g_{n+1}$ develops as

$$
\begin{aligned}
g_{n+1}^{\prime} & =b+V+g_{n+1} \\
& -\sqrt{b^{2}-2 b g_{c}+V^{2}+2 b g_{n}+2 b g_{n+1}} .
\end{aligned}
$$

Setting $g_{n+1}=g$ and $g_{n} \approx V$ the following approximation of $g_{n+1}^{\prime}$ holds,

$$
\begin{aligned}
g_{n+1}^{\prime} & =b+V+g-\sqrt{(b+V)^{2}\left(1+\frac{2 b}{(V+b)^{2}}\left(g-g_{c}\right)\right.} \\
& \approx b+V+g-|b+V|\left(1-\frac{b}{(V+b)^{2}}\left(g-g_{c}\right)\right)
\end{aligned}
$$

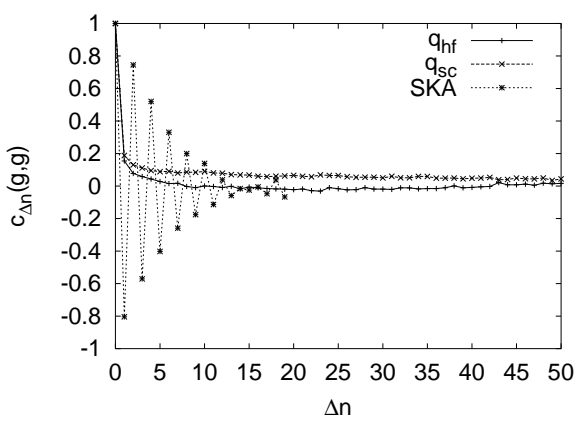

Fig. 11. Correlation function $c_{\Delta n}(g, g)$ for single car data measured at the motorway junction Breitscheidt. Neither the traffic in high flow states $q_{\mathrm{hf}}$ nor in synchronized states $q_{\mathrm{sc}}$ show the strong anticorrelation found in the SKA.

$$
=\left(1-\frac{b}{V+b}\right) g+\frac{b}{V+b} g_{c}
$$

Equation (24) shows, that once $g_{n}=V=$ const, $g$ tends to zero, but is finally stopped at $g_{c}$. A car that starts with $g<g_{c}$ is drawn towards $g_{c}$, which is the fixed-point. Therefore, setting $g_{c}=0$ safe driving can not be assured. This will get even more clear if one determines the minimal gap that occurs over the full range of densities dependent on the stochastic noise strength $\epsilon$ and $g_{c}$, cf. figure 9 On the one hand, it can be seen clearly that the minimal gap found increases with increasing $g_{c}$. On the other hand, the dependence on $\epsilon$ is not so explicit. This results from the fact, that $\epsilon$ does enter the model two-fold. Once, it acts similar as in the SKM (cf. equation (3)) but it also determines $v_{\text {anti. }}$. With respect to the crash-free motion it can nevertheless been stated that there is always a minimal $g_{c}^{*}$ which assures safe driving if $g_{c} \geq g_{c}^{*}$ is chosen. But, the value of $g_{c}^{*}$ depends on the system parameters in a complicated way. It has to be determined by simulation.

A closer look at the correlation function between two arbitrary observables $\xi$ and $\chi$ at car $n, n+\Delta n$ respectively,

$$
c_{\Delta n}\left(\xi_{n}, \chi_{n+\Delta n}\right)=\frac{\left\langle\left(\xi_{n}-\left\langle\xi_{n}\right\rangle\right)\left(\chi_{n+\Delta n}-\left\langle\chi_{n+\Delta n}\right\rangle\right)\right\rangle}{\sigma_{\xi}^{n} \sigma_{\chi}^{n+\Delta n}}(25)
$$

finally explains the effects of anticipation on the system's state. In equation (25) $\sigma_{\xi}^{n}$ stands for the standard deviation of observable $\xi$ taken at car $n$.

In figure 10 the correlation functions $c_{1}(g, g)$ and $c_{0}(g, v)$ are shown. In contrast to the $\mathrm{SKM}^{2}$ there exist a platoon of cars behind the leading car which display a strong anticorrelation between two consecutive gaps, cf. equation (23). Note, that this structure is not destroyed if the lead car also drives stochastically around $\left\langle v_{\text {leader }}\right\rangle$. In the regime of strong anticorrelation $c_{0}(g, v) \approx 0$ a car is somehow free to choose a gap $g$ independent of the speed of the leading car. Therefore, this state reminds one of synchronized flow 1 20]21 22, a traffic state that mostly occurs at bottlenecks. However, further exploration of the simulated data show that the velocity only displays fluctuations of

${ }^{2}$ In $\operatorname{SKM~} c_{1}(g, g) \approx 1$. The same holds for $c_{0}(g, v)$. 
strength $a / 2$ around its mean speed $\left\langle v_{\text {leader }}\right\rangle$, therefore anticipation alone is not able to generate a synchronized state. Moreover, the strong anticorrelation found in the SKA is not present in measured data, cf. figure 11

\section{Conlusions}

The effects of anticipatory driving are investigated by means of simulation as well as analytical calculations. As a reference for comparison a well-understood traffic flow model [8, 9.10] was enhanced by next-nearest-neighbour interaction and investigated under certain aspects. Simulation results show that the introduction of anticipation does not lead to changes in the mechanism that generates wide moving jams nor does the dynamics inside a jam changes. However, one observes a stabilization of the flow in dense traffic, which is "crucial to overcome the difficulties in describing the empirically observed phases and their transitions" 23. Moreover, as one could have been expecting, the "optimized" driving strategy leads to very short temporal headways under free flow conditions. Such short headways are also found in measurements on streets. Their existence can be explained quite general by exploring the mechanism that is introduced in the model by next-nearestneighbour interaction.

In the present article it is shown that this general mechanism works by coupling three cars together to share their two respective headways: if one car is fairly close to the car in front, then its follower has to hold a distance that is roughly equal to the average speed of the car-ensemble. Obviously, this can be generalized to many cars in front, leading in principle to a situation that has been envisioned already by the automobile industry: platoons of cars that are electronically coupled to optimize the energy consumption by driving with very small headways at very large speeds. There, this goal is achieved by very small reaction times of the control system, while an approach based on the ansatz chosen for car-following with anticipation just needs communication to more than one car ahead, but more human-like reaction times.

Surprisingly, despite the fact that the approach of sharing headways sounds fairly natural, it is not easy to show that it happens in reality also. Single-car data (figure 11) show no sign of anticorrelation in car headways, whereas all models working with anticipation display clearly. Beside the SKA we investigated the BL-CA [5] as a representative for a cellular-automaton approach in the same way in order to confirm that assertion. Therefore, anticipation alone is not able to explain all traffic states and their microscopic behaviour found in observations.

The authors like to thank Andreas Schadschneider for useful discussions.

\section{References}

1. B. S. Kerner, Phys. Rev. Lett. 813797 (1998).

2. C. F. Daganyo, M. J. Cassidy, R. L. Bertini, Transp. Res. A 33, 365 (1999).
3. D. Chowdhury, L. Santen, A. Schadschneider, Phys. Rep. 329, 199 (2000).

4. D. Helbing, Rev. Mod. Phys. 73, 1067 (2001).

5. W. Knospe, L. Santen, A. Schadschneider, M. Schreckenberg, J. Phys. A 33477 (2000).

6. R. Jiang, Q.-S. Wu, J. Phys. A 36381 (2003)

7. W. Knospe, L. Santen, A. Schadschneider, M. Schreckenberg, CA models for traffic flow: How do they reproduce the reality?, in preparation.

8. S. Krauss, P. Wagner, C. Gawron, Phys. Rev. E 54, 3707 (1996); 55, 5597 (1997).

9. S. Krauss, PhD thesis, DLR-Forschungsbericht 98-08 (available from www.zaik.de/paper).

10. S. Krauss, in:Traffic and Granular Flow'97, p. 269, M. Schreckenberg, D.E. Wolf (eds.), Springer (1998).

11. P.G. Gipps, Trans. Res. B, 15, 105 (1981).

12. K. Nagel, M. Schreckenberg, J. Physique I2, 2221 (1992).

13. K. Nagel, C. Kayatz, P. Wagner, in:Traffic and Granular Flow'01, Y. Sugiyama et al. (eds.), Springer (2002), eprint cond-mat/0112116

14. R. Barlovic, L. Santen, A. Schadschneider, M. Schreckenber, Eur. Phys. J. 5, 793 (1998).

15. M. Koshi, M. Iwasaki, I. Okuhra, in:Proceedings of the 8th International Symposium on Transportation and Traffic Theory, V.F. Hurdle, E. Hauser, G.N. Steward (eds.), University of Toronto Press, Toronto, Ontario (1983).

16. M. Bando, K. Hasebe, A. Nakayama, S. Shibata, Y. Sugiyama, Jpn. J. Indust. Appl. Math. II, 203 (1994).

17. L. Neubert, L. Santen, A. Schadschneider, M. Schreckenberg, Phys. Rev. E 60, 6480 (1999).

18. W. Knospe, L. Santen, A. Schadschneider, M. Schreckenberg, Phys. Rev. E 65, 056133 (2002).

19. B. Tilch, D. Helbing, in:Traffic and Granular Flow'99, D. Helbing et al. (eds.), Springer (2000).

20. B.S. Kerner, H. Rehborn, Phys. Rev. E 53, R4275 (1996)

21. B.S. Kerner, Phys. World 1225 (1999)

22. B.S. Kerner, Networks Spatial Economics 135 (2001)

23. W. Knospe, L. Santen, A. Schadschneider, M. Schreckenberg, Phys. Rev. E 65, 015101(R) (2002). 